\title{
Sums over Numbers with Restricted Prime Factors
}

\author{
Khadija Mbarki ${ }^{1}$, Walid Wannes ${ }^{2, *}$ \\ ${ }^{1}$ Department of Mathematics, Faculty of Sciences of Monastir, Tunisia \\ ${ }^{2}$ Faculté des sciences de Sfax, BP 1171, Sfax 3000, Tunisie \\ *Corresponding author: wannes.walid.fss@gmail.com
}

Received May 12, 2017; Revised June 30, 2107; Accepted July 12, 2017

\begin{abstract}
Let $q \geq 2$ be a positive integer and $S_{q}(n)$ be the sum of the digits in basis $q$ of the positive integer $n$. We prove that the quotient $\frac{\tilde{\Omega}(n)}{\tilde{\omega}(n)}$ has a normal order one, where $\tilde{\omega}(n)$ and $\tilde{\Omega}(n)$ are respectively, the number of distinct prime factors and the number of prime factors p of a positive integer n counted with multiplicity such that $S_{q}(n) \equiv a \bmod b(a, b \in \mathbb{Z}, b \geq 2)$. Moreover, we discuss sums of the form $\sum_{n \leq x} f(n) \tilde{\omega}(n)$, where $f$ is a
\end{abstract} multiplicative function.

Keywords: sum-of-digits function, uniform distribution modulo1, multiplicative function

Cite This Article: Khadija Mbarki, and Walid Wannes, "Sums over Numbers with Restricted Prime Factors." Turkish Journal of Analysis and Number Theory, vol. 5, no. 5 (2017): 153-158. doi: 10.12691/tjant-5-5-2.

\section{Introduction}

Let $q \geq 2$ be a fixed integer. Every positive integer $n$ has a unique $q$-adic expansion of the form

$$
n=\sum_{j=0}^{v} n_{j} q^{j}, n_{j} \in\{0,1, \ldots, q-1\}
$$

with $n_{v} \neq 0$. For $n=0$, we admit to set $v=0$. The sum of digits function in basis $q$ is defined by

$$
S(n)=S_{q}(n)=n_{0}+n_{1}+\ldots+n_{v} .
$$

The function $S$ is one of the most natural examples of $q$-additive functions (i.e. $S\left(a q^{j}+b\right)=S(a)+S(b)$, with $\left.(a, b, j) \in \mathbb{N}^{3}, \quad 0 \leq b \leq q^{j}\right)$ and $\mathbb{N}$ is the set of non negative integers. Such functions were introduced by Gelfond [7] and further studied by Coquet [1], Kátai [9] and others.

For every $a \in \mathbb{N}$ and $b \geq 2$ such that $(b, q-1)=1$, we define $\tilde{\omega}(n)$ as the number of distinct prime factors $p$ of $n$ such that $S(p) \equiv a \bmod b$ and $\tilde{\Omega}(n)$ the number of distinct prime factors of $n$ counted with multiplicity such that $S(p) \equiv a \bmod b$. Therefore,

$$
\tilde{\omega}(n)=\tilde{\omega}_{a, b, q}(n)=\sum_{\substack{p \mid n \\ S(p) \equiv a \bmod b}} 1
$$

$$
\text { and } \tilde{\Omega}(n)=\tilde{\Omega}_{a, b, q}(n)=\sum_{\substack{p^{\alpha} \mid n \\ S(p) \equiv a \bmod b}} 1
$$

Both functions have been studied in [12,13], it was proved that their normal order is $\frac{1}{b} \log \log n$. A crucial part in the proof is the following estimation

$$
\sum_{\substack{p \leq x \\ S(p) \equiv a \bmod b}} \frac{1}{p}=\frac{1}{b} \log \log x+\beta+O\left(\frac{1}{\log x}\right),
$$

where

$$
\beta=\sum_{1 \leq j \leq b-1} e\left(\frac{-a j}{b}\right) \int_{2}^{+\infty}\left(\sum_{p \leq t} e\left(S(p) \frac{j}{b}\right)\right) \frac{d t}{t^{2}} .
$$

The aim of this paper is to provide asymptotic formulas for sums involving $\tilde{\omega}(n)$ and $\tilde{\Omega}(n)$. We consider firstly the sum

$$
\sum_{n \leq x} \frac{\tilde{\Omega}(n)}{\tilde{\omega}(n)}
$$

Then, by using elementary methods, we discuss sums of the form

$$
\sum_{n \leq x} f(n) \tilde{\omega}(n)
$$

where $f$ is one of the classical arithmetic functions $\mu, \varphi$ and $\sigma$, where $\mu$ is the Möbius function, while $\varphi$ and $\sigma$ 
are respectively, the Euler function and the sum of divisors function. This extends the results known through the work of De Koninck [4], De Koninck and Sitaramachandrarao [5] to primes verifying a digital constraint. Finally, we give some related results about the distribution of $\tilde{\Omega}(n)-\tilde{\omega}(n)$ and uniform distribution modulo 1 of sequences involving $\tilde{\Omega}(n)$ and $\tilde{\omega}(n)$.

Throughout this paper, $p$ always denotes a prime number. For any real $x$, we set $e(x)=e^{2 \pi i x}$. The notation $(a, b)$ refers to the greatest common divisor of $a$ and $b$. $\omega(n)$ is the number of distinct prime factors of $n$ while $\Omega(n)$ is the number of prime factors counted with multiplicity of the integer $n$. We recall that the notation $U \ll V$ is equivalent to the statement that $U=O(V)$ for positive functions $U$ and $V$ and the implied constants in the symbols " $O$ ", “ «" are absolute. We also use the symbol " $O$ " with its usual meaning, the statement $U=o(V)$ is equivalent to $U / V \rightarrow 0$.

\section{The Sum of Quotients}

Since $\omega(n)$ and $\Omega(n)$ have both the normal order $\log \log n$ (see [8]), it is obvious that $\frac{\Omega(n)}{\omega(n)}$ has normal order one which is shown by De Koninck in [4]. A crucial part in his proof is an elementary estimation of $\sum_{2 \leq n \leq x} \frac{1}{\omega(n)}$. In this part, we consider an analogue problem, since $\tilde{\omega}(n)$ and $\tilde{\Omega}(n)$ have both the normal order $\frac{1}{b} \log \log n$ (see [12]). So, we put

$$
\Delta(n)=\left\{\begin{array}{l}
\frac{\tilde{\Omega}(n)}{\tilde{\omega}(n)}, \text { if } \tilde{\omega}(n) \neq 0, \\
1, \text { otherwise. }
\end{array}\right.
$$

Then, the average value of $\Delta(n)$ is given in the following theorem.

Theorem 2.1.

$$
\sum_{n \leq x} \Delta(n)=x+O\left(\frac{x}{\log \log x}\right)
$$

Proof. Our first task is to estimate $\sum_{\substack{n \leq x \\ \tilde{\omega}(n) \neq 0}} \frac{1}{\tilde{\omega}(n)}$. Indeed, we have

$$
\begin{aligned}
& \sum_{\substack{n \leq x \\
\tilde{\omega}(n) \neq 0}} \frac{1}{\tilde{\omega}(n)} \\
= & \sum_{\substack{n \leq x \\
0<2 b \tilde{\omega}(n)<\log \log x}} \frac{1}{\tilde{\omega}(n)}+\sum_{\substack{n \leq x \\
2 b \tilde{\omega}(n) \geq \log \log x}} \frac{1}{\tilde{\omega}(n)}
\end{aligned}
$$

$$
\leq \sum_{\substack{n \leq x \\ 2 b \tilde{\omega}(n)<\log \log x}} 1+2 b \frac{x}{\log \log x} .
$$

Proposition 2.2 of [12] implies that

$$
\sum_{n \leq x}\left(\tilde{\omega}(n)-\frac{1}{b} \log \log x\right)^{2} \leq C x \log \log x
$$

where $C$ is an absolute constant. So,

$$
\sum_{\substack{n \leq x \\ 2 b \tilde{\omega}(n)<\log \log x}}\left(\tilde{\omega}(n)-\frac{1}{b} \log \log x\right)^{2} \leq C x \log \log x .
$$

Or,

$$
\begin{aligned}
& \frac{1}{4 b^{2}}(\log \log x)^{2} \sum_{\substack{n \leq x \\
2 b \tilde{\omega}(n)<\log \log x}} 1 \\
& \leq \sum_{\substack{n \leq x \\
2 b \tilde{\omega}(n)<\log \log x}}\left(\tilde{\omega}(n)-\frac{1}{b} \log \log x\right)^{2} .
\end{aligned}
$$

Then,

$$
\sum_{\substack{n \leq x \\ 2 b \tilde{\omega}(n)<\log \log x}} 1 \leq 4 b^{2} C \frac{x}{\log \log x} .
$$

So, we obtain

$$
\sum_{\substack{n \leq x \\ \tilde{\omega}(n) \neq 0}} \frac{1}{\tilde{\omega}(n)}=O\left(\frac{x}{\log \log x}\right) .
$$

Now, we use (2.2) to estimate the left hand side of (2.1) as follows

$$
\begin{aligned}
& \sum_{n \leq x} \Delta(n)=\sum_{\substack{n \leq x \\
\tilde{\omega}(n)=0}} \Delta(n)+\sum_{\substack{n \leq x \\
\tilde{\omega}(n) \neq 0}} \Delta(n) \\
& =\sum_{\substack{n \leq x \\
\tilde{\omega}(n)=0}} \Delta(n)+\sum_{\substack{n \leq x \\
\tilde{\omega}(n) \neq 0}} \sum_{\substack{p^{m_{\mid n}} \\
S(p) \equiv a \bmod b}} \frac{1}{\tilde{\omega}(n)} \\
& =\sum_{\substack{n \leq x \\
\tilde{\omega}(n)=0}} \Delta(n)+\sum_{\substack{n \leq x \\
\tilde{\omega}(n) \neq 0}} \sum_{\substack{p \mid n \\
S(p) \equiv a \bmod b}} \frac{1}{\tilde{\omega}(n)} \\
& +\sum_{\substack{n \leq x \\
\tilde{\omega}(n) \neq 0}} \sum_{\substack{p^{m} \mid n, m \geq 2 \\
S(p) \equiv a \bmod b}} \frac{1}{\tilde{\omega}(n)} \\
& =\sum_{\substack{n \leq x \\
\tilde{\omega}(n) \neq 0}} 1+\sum_{\substack{n \leq x \\
\tilde{\omega}(n) \neq 0}} 1+\sum_{\substack{p^{m} \leq x, m \geq 2 \\
S(p) \equiv a \bmod b}} \sum_{n \leq x p} \frac{1}{\tilde{\omega}\left(n p^{m}\right)} \\
& =\sum_{n \leq x} 1+O\left(\sum_{\substack{p^{m} \leq x, m \geq 2 \\
s(p) \equiv a \bmod b}} \sum_{\substack{n \leq x p^{-m} \\
\tilde{\omega}(n) \neq 0}} \frac{1}{\tilde{\omega}(n)}\right)
\end{aligned}
$$




$$
\begin{aligned}
& =x+O\left(\sum_{p^{m_{\leq x, m \geq 2}}} \sum_{\substack{n \leq x p \\
\tilde{\omega}(n) \neq 0}} \frac{1}{\tilde{\omega}(n)}\right) \\
& =x+O\left(\sum_{\substack{n \leq x \\
\tilde{\omega}(n) \neq 0}} \sum_{\substack{m_{\leq \frac{x}{n}} \\
m \geq 2}} \frac{1}{\tilde{\omega}(n)}\right)
\end{aligned}
$$

Since $\sum_{\substack{p^{m} \leq t \\ m \geq 2}} 1=O\left(t^{\frac{1}{2}}\right)$, then by partial summation, we can write

$$
\begin{aligned}
& \sum_{\substack{n \leq x \\
\tilde{\omega}(n) \neq 0}} \sum_{\substack{m \leq \frac{x}{n} \\
m \geq 2}} \frac{1}{\tilde{\omega}(n)} \leq \sum_{\substack{n \leq x \\
\tilde{\omega}(n) \neq 0}} \sum_{\substack{m_{\leq} \frac{x}{n} \\
m \geq 2}} 1 \\
& \ll \sqrt{x} \sum_{\substack{n \leq x \\
\tilde{\omega}(n) \neq 0}} \frac{1}{\sqrt{n} \tilde{\omega}(n)} \\
&=\sqrt{x}\left(x^{-\frac{1}{2}} \sum_{\substack{n \leq x \\
\tilde{\omega}(n) \neq 0}} \frac{1}{\tilde{\omega}(n)}+\frac{1}{2} \int_{1}^{x} t^{-\frac{3}{2}} \sum_{\substack{n \leq t \\
\tilde{\omega}(n) \neq 0}} \frac{1}{\tilde{\omega}(n)} d t\right) \\
&= O\left(\frac{x}{\log \log x}\right),
\end{aligned}
$$

where the last equality is derived from (2.2). Assembling (2.3) and (2.4), we obtain

$$
\sum_{n \leq x} \Delta(n)=x+O\left(\frac{x}{\log \log x}\right),
$$

which completes the proof of the theorem.

\section{On the Sum $\sum_{n \leq X} f(n) \tilde{\omega}(n)$}

In this section, we discuss sums of the form $\sum_{n \leq x} f(n) \tilde{\omega}(n)$, where $f(n)$ is either $\mu(n), \varphi(n)$ or $\sigma(n)$. We note that the case $\sum_{n \leq x} f(n) \omega(n)$, where $f$ is a multiplicative function, was discussed in [[6], Chapter 9]. Theorem 3.1. Let $q, b$ be integers $\geq 2$ satisfying $(b, q-1)=1$. Then for any integer $m \geq 1$, there exist constants $E_{i}, \quad 0 \leq i \leq m-1$ such that

$$
\sum_{n \leq x} \mu(n) \tilde{\omega}(n)=\frac{x}{b} \sum_{i=1}^{m} \frac{E_{i-1}}{(\log x)^{i}}+O\left(\frac{x}{(\log x)^{m+1}}\right) .
$$

Proof.

$$
\begin{aligned}
& \sum_{n \leq x} \mu(n) \tilde{\omega}(n)=\sum_{n \leq x} \mu(n) \sum_{\substack{p \mid n \\
S(p) \equiv a \bmod b}} 1 \\
& =\sum_{\substack{p n \leq x \\
S(p) \equiv a \bmod b}} \mu(p n)=-\sum_{\substack{p n \leq x, p \nmid n \\
S(p) \equiv a \bmod b}} \mu(n) \\
& =-\sum_{\substack{p n \leq x \\
S(p) \equiv a \bmod b}} \mu(n)+\sum_{\substack{p n \leq x, p \mid n \\
S(p) \equiv a \bmod b}} \mu(n) \\
& =-\sum_{\substack{p n \leq x \\
S(p) \equiv a \bmod b}} \mu(n)+\sum_{\substack{p^{2} n \leq x \\
S(p) \equiv a \bmod b}} \mu(p n) \\
& =-\sum_{\substack{p n \leq x \\
S(p) \equiv a \bmod b}} \mu(n)-\sum_{\substack{p^{2} n \leq x, p \nmid n \\
S(p) \equiv a \bmod b}} \mu(n) \\
& =-\sum_{\substack{p n \leq x \\
S(p) \equiv a \bmod b}} \mu(n)-\sum_{\substack{p^{2} n \leq x \\
S(p) \equiv a \bmod b}} \mu(n) \\
& +\sum_{\substack{p^{2} n \leq x, p \mid n \\
S(p) \equiv a \bmod b}} \mu(n) .
\end{aligned}
$$

By pursuing the same procedure, we get

$$
\sum_{n \leq x} \mu(n) \tilde{\omega}(n)=-\sum_{\substack{p^{\alpha} n \leq x \\ S(p) \equiv a \bmod b}} \mu(n) .
$$

Now, we write

$$
\begin{aligned}
& \sum_{n \leq x} \mu(n) \tilde{\omega}(n) \\
& =-\sum_{\substack{p n \leq x \\
S(p) \equiv a \bmod b}} \mu(n)-\sum_{\substack{p^{\alpha} n \leq x, \alpha \geq 2 \\
S(p) \equiv a \bmod b}} \mu(n) \\
& =-\Sigma_{1}-\Sigma_{2},
\end{aligned}
$$

say.

For the estimation of $\Sigma_{1}$, we use Dirichlet's hyperbola method and we obtain

$$
\begin{aligned}
& \Sigma_{1}=\sum_{\substack{p n \leq x \\
S(p) \equiv a \bmod b}} \mu(n) \\
& =\sum_{\substack{p n \leq x, p \leq \sqrt{x} \\
S(p) \equiv a \bmod b}} \mu(n)+\sum_{\substack{p n \leq x, n \leq \sqrt{x} \\
S(p) \equiv a \bmod b}} \mu(n) \\
& -\sum_{n \leq \sqrt{x}} \mu(n) \sum_{\substack{p \leq \sqrt{x} \\
S(p) \equiv a \bmod b}} 1 \\
& =\Sigma_{1}^{(1)}+\Sigma_{1}^{(2)}-\Sigma_{1}^{(3)} .
\end{aligned}
$$

Now, it is well-known (See [15]) that there exists a constant $A>0$ such that

$$
\begin{aligned}
& M(x)=\sum_{n \leq \sqrt{x}} \mu(n) \\
& =O\left(x \exp \left(-A \log ^{\frac{3}{5}} x(\log \log x)^{-\frac{1}{5}}\right)\right) .
\end{aligned}
$$


Hence,

$$
\begin{aligned}
& \Sigma_{1}^{(1)}=\sum_{\substack{p n \leq x, p \leq \sqrt{x} \\
S(p) \equiv a \bmod b}} \mu(n)=\sum_{\substack{p \leq \sqrt{x} \\
S(p) \equiv a \bmod b}} \sum_{\substack{p \leq \frac{x}{p} \\
S(p) \equiv a \bmod b}} \mu(n) \\
& \left.\ll \sum^{\frac{3}{5}} \exp \left(-A \log ^{\frac{x}{p}}\right) \log \log \frac{1}{5}\left(\frac{x}{p}\right)\right) \\
& \ll x \exp \left(-A \log ^{\frac{3}{5}} x\right) \sum_{\substack{p \leq \sqrt{x} \\
S(p) \equiv a \bmod b}} \frac{1}{p} \\
& =O\left(x \log \log x \exp \left(-A \log ^{\frac{3}{5}} x\right)\right)
\end{aligned}
$$

where the last bound is derived from (1.1). For the estimations of $\Sigma_{1}^{(2)}$ and $\Sigma_{1}^{(3)}$, we write

$$
\begin{aligned}
& \Sigma_{1}^{(3)}=\sum_{n \leq \sqrt{x}} \mu(n) \sum_{\substack{p \leq \sqrt{x} \\
S(p) \equiv a \bmod b}} 1 \\
& =\left(\frac{1}{b} \operatorname{Li}(\sqrt{x})+O\left(\sqrt{x}(\log x)^{-c}\right)\right) M(\sqrt{x}) \\
& =\frac{1}{b} \operatorname{Li}(\sqrt{x}) M(\sqrt{x})+O\left(x \frac{\exp \left(-A \log ^{\frac{3}{5}} x\right)}{(\log x)^{c}}\right)
\end{aligned}
$$

for any c $>0$, by using the prime number theorem and [[11], Théorème 3] in the case $(b, q-1)=1$. Here

$$
\begin{aligned}
& \operatorname{Li}(x)=\int_{2}^{x} \frac{d t}{\log t} \text {. Also } \\
& \Sigma_{1}^{(2)}=\sum_{n \leq \sqrt{x}} \mu(n) \sum_{\substack{p \leq \frac{x}{n} \\
S(p) \equiv a \bmod b}} 1 \\
& =\sum_{n \leq \sqrt{x}} \mu(n)\left\{\frac{1}{b} \operatorname{Li}\left(\frac{x}{n}\right)+O\left(\frac{x}{n} \log \left(\frac{x}{n}\right)^{-c}\right)\right\} \\
& =\frac{1}{b} \sum_{n \leq \sqrt{x}} \mu(n) \operatorname{Li}\left(\frac{x}{n}\right)+O\left(x \frac{\exp \left(-A \log ^{\frac{3}{5}} x\right)}{(\log x)^{c}}\right) .
\end{aligned}
$$

However, by partial summation, we derive that

$$
\begin{aligned}
& \frac{1}{b} \sum_{n \leq \sqrt{x}} \mu(n) \operatorname{Li}\left(\frac{x}{n}\right) \\
& =\frac{1}{b} \operatorname{Li}(\sqrt{x}) M(\sqrt{x})+\frac{1}{b} \int_{1}^{\sqrt{x}} \frac{M(t)}{\log (x / t)} \frac{x}{t^{2}} d t \\
& =\frac{1}{b} \operatorname{Li}(\sqrt{x}) M(\sqrt{x})+\frac{x}{b \log x} \int_{1}^{\sqrt{x}} \frac{M(t)}{t^{2}}\left[1-\frac{\log t}{\log x}\right]^{-1} d t .
\end{aligned}
$$

Now,

$$
\begin{aligned}
& \int_{1}^{\sqrt{x}} \frac{M(t)}{t^{2}}\left[1-\frac{\log t}{\log x}\right]^{-1} d t \\
& =\int_{1}^{\sqrt{x}} \frac{M(t)}{t^{2}}\left\{\begin{array}{l}
1+\frac{\log t}{\log x}+\ldots+\left[\frac{\log t}{\log x}\right]^{m-1} \\
\left.+O\left(\frac{\log t}{\log x}\right)^{m}\right)
\end{array}\right\} \\
& =\sum_{i=0}^{m-1} \frac{1}{(\log x)^{i}} \int_{1}^{+\infty} \frac{M(t)(\log t)^{i}}{t^{2}} d t+O\left(\frac{1}{(\log x)^{m}}\right)
\end{aligned}
$$

From (3.7), (3.8) and (3.9), we deduce

$$
\begin{aligned}
& \Sigma_{1}^{(2)}=\frac{1}{b} \operatorname{Li}(\sqrt{x}) M(\sqrt{x}) \\
& +\frac{x}{b} \sum_{i=1}^{m} \frac{1}{(\log x)^{i}} \int_{1}^{+\infty} \frac{M(t)(\log t)^{i-1}}{t^{2}} d t+O\left(\frac{x}{(\log x)^{m+1}}\right) .
\end{aligned}
$$

Assembling (3.5), (3.6) and the last equality, one can show that

$$
\Sigma_{1}=\frac{x}{b} \sum_{i=1}^{m} \frac{E_{i-1}}{(\log x)^{i}}+O\left(\frac{x}{(\log x)^{m+1}}\right)
$$

with

$$
E_{i}=\int_{1}^{+\infty} \frac{M(t)(\log t)^{i}}{t^{2}} d t
$$

For $\alpha \geq 2$, by using (3.4), we get

$$
\begin{aligned}
& \Sigma_{2}=\sum_{\substack{p^{\alpha} n \leq x \\
S(p) \equiv a \bmod b}} \mu(n)=\sum_{\substack{p \leq x \\
s(p) \equiv a \bmod b}} \sum_{\substack{\alpha \\
n \leq \frac{x}{p^{\alpha}}}} \mu(n) \\
& \ll \sum_{\substack{p \leq x \\
S(p) \equiv a \bmod b}}^{\frac{1}{\alpha}} \frac{x}{p^{\alpha}} \exp \left(\times\left(\log \log \left(\frac{x}{p^{\alpha}}\right)\right)^{-\frac{1}{5}}\right) \\
& \ll x \exp \left(-A \log ^{\frac{3}{5}} x\right) \sum_{\substack{p \leq x \\
\frac{1}{\alpha} \\
S(p) \equiv a \bmod b}} \frac{1}{p^{\alpha}} \\
& =O\left(x \exp \left(-A \log ^{\frac{3}{5}} x\right)\right) \text {. }
\end{aligned}
$$

Finally, formulas (3.2), (3.10) and (3.11) give the desired estimation.

In order to provide a corresponding result for $\varphi$ and $\sigma$, we need the lemma below, which can be proved by the same method as in the proof provided in [13]. 
Lemma 3.2. Let $i \geq 1$ and $a_{i}=\frac{(i-1) !}{2^{i}}$. Then, for any integer $N \geq 1$ we have

$$
\sum_{\substack{p \leq x \\ S(p) \equiv a \bmod b}} p=\frac{x^{2}}{b} \sum_{i=1}^{N} \frac{a_{i}}{\log ^{i} x}+O_{b}\left(\frac{x^{2}}{\log ^{N+1} x}\right) .
$$

Theorem 3.3. Let $q, b$ be integers $\geq 2$ verifying $(b, q-1)=1$. Then for any integer $m \geq 1$, there exist constants $K_{1}, K_{2}, F_{i}, G_{i}, 0 \leq i \leq m-1$ such that

$$
\begin{aligned}
& \sum_{n \leq x} \varphi(n) \tilde{\omega}(n)=\frac{3}{b \pi^{2}} x^{2} \log \log x \\
& +\frac{x^{2}}{b} \sum_{i=1}^{m-1} \frac{F_{i}}{(\log x)^{i}}+K_{1} x^{2}+O\left(\frac{x^{2}}{(\log x)^{m}}\right) . \\
& \sum_{n \leq x} \sigma(n) \tilde{\omega}(n)=\frac{\pi^{2}}{12 b} x^{2} \log \log x \\
& +\frac{x^{2}}{b} \sum_{i=1}^{m-1} \frac{G_{i}}{(\log x)^{i}}+K_{2} x^{2}+O\left(\frac{x^{2}}{(\log x)^{m}}\right) .
\end{aligned}
$$

Proof. For the Euler function, if we proceed as in (3.1), we obtain

$$
\sum_{n \leq x} \varphi(n) \tilde{\omega}(n)=\sum_{\substack{p^{\alpha} n \leq x \\ S(p) \equiv a \bmod b}}(p-1) \varphi(n)
$$

Since $\sigma$ is a multiplicative function verifying $\sigma\left(p^{2}\right)+p=\sigma^{2}(p)$, one can show that

$$
\begin{aligned}
& \sum_{n \leq x} \sigma(n) \tilde{\omega}(n) \\
& =\sum_{\substack{p n \leq x \\
S(p) \equiv a \bmod b}}(p+1) \sigma(n) \\
& -\sum_{\substack{p^{2} n \leq x \\
S(p) \equiv a \bmod b}} p(p+1) \sigma(n) .
\end{aligned}
$$

By using the same approach as in Theorem 3.1, Lemma 3.2 and the following well known formulas

$$
\begin{aligned}
& \sum_{n \leq x} \varphi(n)=\frac{3}{\pi^{2}} x^{2}+O(x \log x), \\
& \sum_{n \leq x} \sigma(n)=\frac{\pi^{2}}{12} x^{2}+O(x \log x)
\end{aligned}
$$

combined with (3.12) and (3.13), we get the result.

\section{Other Results}

\subsection{The Distribution of $\tilde{\Omega}(n)-\tilde{\omega}(n)$}

Rényi proved that for any positive integer $k$, the set of numbers $\mathrm{n}$ such that $\Omega(n)-\omega(n)=k$ has density $d_{k}$, where $d_{k}$ are the power series coefficients of the meromorphic function

$$
F(z)=\sum_{k=0}^{+\infty} d_{k} z^{k}=\prod_{p}\left(1-\frac{1}{p}\right)\left(1+\frac{1}{p-z}\right)
$$

Let $N_{k}(x)$ denote the number of $n \leq x$ for which $\Omega(n)-\omega(n)=k$. In [[14], Theorem 2.16], Montgomery and Vaughan showed a quantitative form of Rényi's theorem which states that for any nonnegative integer $k$ and any $x \geq 2$, one has

$$
N_{k}(x)=d_{k} x+O\left(\left(\frac{3}{4}\right)^{k} \sqrt{x}(\log x)^{4 / 3}\right)
$$

where

$$
d_{k}=\frac{6}{\pi^{2}} \sum_{\substack{m \in \mathcal{M} \\ \Omega(m)-\omega(m)=k}} \frac{1}{m} \prod_{p \mid m}\left(1+\frac{1}{p}\right)^{-1}
$$

and $\mathcal{M}$ is the set of powerful numbers i.e., those m such that $p\left|m \Rightarrow p^{2}\right| m$. In this section, we will study the distribution of the function $\tilde{\Omega}(n)-\tilde{\omega}(n)$. An analogous formula to (4.1) holds for the number of $n \leq x$ for which $\tilde{\Omega}(n)-\tilde{\omega}(n)=k$. Denote $\tilde{N}_{k}(x)$ this number. By following the same steps as in the proof of [[14], Theorem 2.16], we get the following result.

Proposition 4.1. For any nonnegative integer $k$ and any $x \geq 2$,

$$
\tilde{N}_{k}(x)=\tilde{d}_{k} x+O\left(\left(\frac{3}{4}\right)^{k} \sqrt{x}(\log x)^{4 / 3}\right)
$$

where

$$
\tilde{d}_{k}:=\frac{6}{\pi^{2}} \sum_{\substack{m \in \mathcal{M} \\ \Omega(m)-\tilde{\omega}(m)=k}} \frac{1}{m} \prod_{p \mid m}\left(1+\frac{1}{p}\right)^{-1}
$$

and $\mathcal{M}$ is the set defined above.

\subsection{Uniform Distribution Modulo 1}

We denote by $\mathcal{M}_{1}$ the set of multiplicative arithmetical functions $f$ verifying $|f(n)| \leq 1(n \in \mathbb{N})$.

H. Daboussi proved that for every irrational $(\alpha \in \mathbb{R})$, uniformly for $f$ in $\mathcal{M}_{1}$, we have

$$
\frac{1}{x} \sum_{n \leq x} f(n) e(n \alpha) \rightarrow 0 .
$$

The proof is given in his paper [2] written jointly with H. Delange.

An immediate consequence of Daboussi's result is the following: If $\alpha$ is an irrational number and $g$ is a real valued additive arithmetical function, then the sequence $(g(n)+\alpha n)_{n \in \mathbb{N}}$ is uniformly distributed modulo 1 and 
this follows from Weyl's criterion (see [[10], Theorem 5.6]). Since $\tilde{\Omega}(n)$ and $\tilde{\omega}(n)$ are both additive real valued arithmetical functions then, for any irrational number $\alpha$ the following sequences $(\tilde{\omega}(n)+\alpha n)_{n \in \mathbb{N}}$ and $(\tilde{\Omega}(n)+\alpha n)_{n \in \mathbb{N}}$ are uniformly distributed modulo 1 .

\section{References}

[1] J. Coquet. Sur les fonctions Q-multiplicatives et Q-additives, Thèse $3^{\text {ème }}$ cycle, Orsay, (1975).

[2] H. Daboussi, H. Delange. On multiplicative arithmetical functions whose module does not exceed one, J. London Math. Soc. 26, p.245-264, (1982).

[3] M. Drmota, C. Mauduit, J. Rivat. Primes with an average sum of digits, Compositio Math. 145, p.271-292, (2009).

[4] J. M. De Koninck. Sums of quotients of additive functions, Proc. Amer. Math. Soc, (44), p. 35-38, (1974).

[5] J. M. De Koninck and R. Sitaramachandrarao. Sums involving the largest prime divisor of an integer II, Indian J. pure appl. Math., (19), p. 990-1004, (1988).
[6] J. M. De Koninck and A. Ivić, Topics in Arithmetical functions Notas de Matematica 72, North Holland, Amsterdam, (1980).

[7] A. O. Gelfond. Sur les nombres qui ont des propriétés additives et multiplicatives données, Acta Arith. (13), p. 259-265, (1968).

[8] G.H. Hardy and E. M. Wright. An introduction to the theory of numbers, Oxford University Press, (1979).

[9] I. Kàtai. Distribution of q-additive functions, in "Probability Theory and Applications" Kluwer Academic, Dordrecht, p. 309-318, (1992).

[10] L. Kuipers, H. Niederreiter. Uniform distribution of sequences John Willey, New York, (1974).

[11] C. Mauduit, J.Rivat. Sur un problème de Gelfond: la somme des chiffres des nombres premiers, Ann. of Math. 71, p.159-1646, (2010).

[12] M. Mkaouar, W. Wannes. On the number of restricted prime factors of an integer, Acta Mathematica Hungarica, (143), p. 8895, (2014).

[13] M. Mkaouar, W. Wannes. On the normal number of prime factors of $\varphi(n)$ subject to certain congruence conditions, J. of Number Theory (160), p. 629-645, (2016).

[14] H.L. Montgomery, R.C. Vaughan. Multiplicative Number Theory I. Classical Theory Cambridge Studies in advanced Mathematics 97.

[15] A. Walfisz. Weylsche Exponentialsummen in der neuren Zahlentheorie, Berlin, (1963). 